\title{
Directional dark matter search with the NEWSdm experiment
}

\author{
Giovanni De Lellis ${ }^{1,2, a}$ \\ ${ }^{1}$ Dipartimento di Fisica "E. Pancini", Università "Federico II" di Napoli, Naples, Italy \\ ${ }^{2}$ Istituto Nazionale di Fisica Nucleare, Naples, Italy
}

\begin{abstract}
The nature of Dark Matter is one of the fundamental questions to be answered. Direct Dark Matter searches are focussed on the development, construction, and operation of detectors looking for the scattering of Weakly Interactive Massive Particles (WIMPs) with target nuclei. The measurement of the direction of WIMP-induced nuclear recoils is a challenging strategy to extend dark matter searches beyond the neutrino floor and provide an unambiguous signature of the detection of Galactic dark matter. Current directional experiments are based on the use of gas TPC whose sensitivity is strongly limited by the small achievable detector mass. NEWSdm is an innovative directional experiment proposal based on the use of a solid target made by newly developed nuclear emulsion films and read-out systems achieving a position accuracy of $10 \mathrm{~nm}$.
\end{abstract}

\section{Introduction}

Current experimental efforts for direct Dark Matter searches are devoted to the search for rare interactions between galactic halo WIMPs and nuclei with detectors located in underground laboratories. Since dark matter detectors are rapidly improving their sensitivity, in the next decade they will encounter the neutrino background where Solar, atmospheric, and diffuse supernova neutrinos mimic the dark matter signal. Neutrinos are the ultimate background for these searches as they produce recoils with similar rates and energy spectra. Moreover, the controversy in the low-mass (order of 10 $\mathrm{GeV} / \mathrm{c}^{2}$ ) WIMP region where some dark matter hints were shown, highlights the need for additional discrimination power between WIMP events and backgrounds to unambiguously prove WIMP signals. New generation detectors capable of measuring the direction of nuclear recoil tracks resulting from the WIMP elastic scattering off target nuclei would provide the discrimination of neutrino background and the unambiguous identification of galatic WIMPs as dark matter candidates.

Several approaches have been proposed [1]. The use of a solid target is the only approach to achieve large masses and therefore high sensitivity in the low cross-section region. Nevertheless, in a solid medium, the track length of a nuclear recoil would be of the order of a few hundred nanometers, thus requiring a detector with ultra-high tracking resolution. This is the challenge addressed by the NEWSdm proposal [2].

The NEWSdm apparatus consists of a nuclear emulsion detector acting both as target and as nanometric tracking device. Nuclear emulsions are made of silver halide crystals embedded in a gelatine matrix. When ionizing particles pass through it, some of the halide crystals are modified

\footnotetext{
a e-mail: giovanni.de.lellis@ cern.ch
} 
in such a way that they are turned into grains of silver after the developing process. The threedimensional trajectory can be reconstructed with an optical microscope by connecting all the silver grains. NEWSdm employs a novel emulsion technology called Nano Imaging Trackers (NIT) [3, 4] with $\mathrm{AgBr}$ crystals of nanometric size, one order of magnitude smaller than those used in the OPERA experiment [5]. The detector is a bulk of NIT surrounded by a shield to reduce the external background. It is placed on an equatorial telescope to absorb the Earth rotation, thus keeping its orientation fixed towards the apparent WIMP flux. The angular distribution of the WIMP-scattered nuclei is therefore expected to be strongly anisotropic.

\section{Optical microscope read-out system}

NIT have a linear density of about 11 crystals/ $\mu \mathrm{m}$ [3], thus making the reconstruction of trajectories with path lengths shorter than $100 \mathrm{~nm}$ possible, if analyzed by means of microscopes with enough resolution. The search for signal candidates requires the scanning of the whole emulsion volume. The read-out system has therefore to fulfill two main requirements: a fast, completely automated, scanning system is needed to analyse the target volume over a time scale comparable with the exposure; the spatial resolution has to reach the challenging value of a few tens of nanometers, well beyond the diffraction limit.

The NIT analysis is performed with a two-step approach: a fast scanning with a state-of-the-art resolution for the signal preselection followed by a pin-point check of preselected candidates with unprecedented nanometric resolution. A fast scanning is performed at first with an improved version of the optical microscope used for OPERA films [6]. An R\&D program has achieved a speed of about $200 \mathrm{~cm}^{2} / \mathrm{h}[7,8]$. Given the intrinsic resolution of the optical microscope $(\sim 200 \mathrm{~nm})$, the sequence of several grains making a track of a few hundred nanometers may appear as a single cluster. Nevertheless, a cluster made of several grains tends to have an elliptical shape with the major axis along the direction of the trajectory, while a cluster produced by a single grain tends to have a spherical shape. The shape analysis with an elliptical fit is indeed the first approach to select signal.

In order to reproduce the effect of a WIMP-induced nuclear recoil and to measure the efficiency and the resolution of the new optical prototype, a test beam with low velocity ions was performed. $\mathrm{Kr}$ ion beam with energies of 200 and $400 \mathrm{keV}$ [9] and a $\mathrm{C}$ ion beam with energies of 60, 80 and 100 $\mathrm{keV}$ were used. Silver grains belonging to the tracks appear as a single cluster. An elliptical fit of the cluster shape allows a clear separation between fog grains and signal tracks [10].

The second step of the analysis exploits the plasmon resonance effect occurring when nanometric silver grains are dispersed in a dielectric medium [11]. The polarization dependence of the resonance reflects the shape anisotropy and can be used to infer the presence of non-spherical nanometric silver grains within a cluster made of several grains. This effect is used to retrieve track information beyond the diffraction limit. Images of the same cluster taken with different polarization angles show a displacement of the position of its barycenter. The analysis of this displacement allows to distinguish clusters made of a single grain from those made of two or more grains. An unprecedented accuracy better than $10 \mathrm{~nm}$ has been achieved in both coordinates with this method as shown in Fig. 1. The clusters showing a barycenter displacement larger than $3 \sigma$ are defined as "moving grains" and those below are defined as "static grains". Moreover, when more than one grain is reconstructed, the corresponding track is called micro-track. The plot in Fig. 2 shows the angular distribution for these three different components produced by the implantation of $100 \mathrm{keV}$ Carbon ions: static grains are uniformly distributed as expected since they are mostly due to isolated grains produced by thermal excitation, while a clear angular peak along the direction of the implantation is shown for moving grains. Micro-tracks are also produced by implanted carbon ions when their track lengths exceeds 
about $200 \mathrm{~nm}$ and they also show an angular peak. This plot demonstrates the directionality of the emulsion technique.

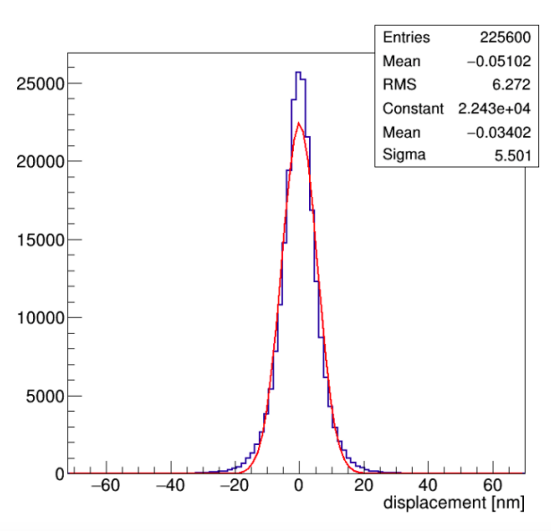

Figure 1. Achieved position accuracy better than $10 \mathrm{~nm}$.

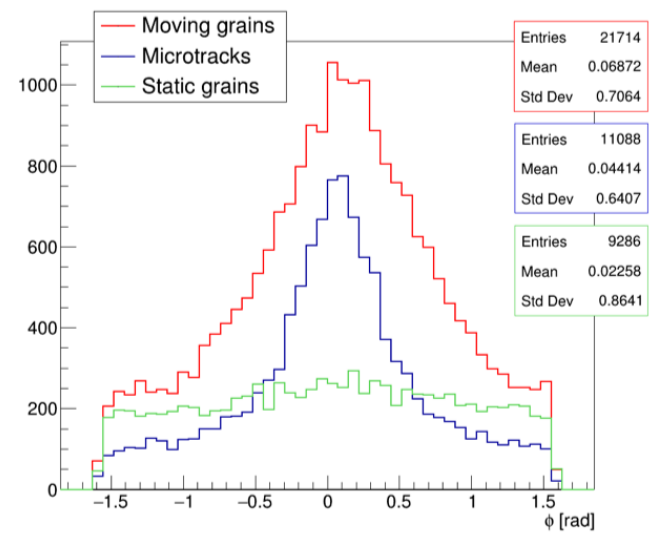

Figure 2. Angular distribution of single grains and micro-tracks.

\section{Experimental setup}

NEWSdm has setup at Gran Sasso a facility for the emulsion handling and film production. A dedicated structure was constructed to shield a detector of $10 \mathrm{~g}$ mass against the environmental background sources over an exposure time of about 1 month. The aim is to measure the detectable background and validate estimates from simulations. This test will pave the way for a pilot experiment with an exposure of about $10 \mathrm{~kg}$ year. The experimental setup is located in the Hall B of the Gran Sasso Laboratory and it consists of a shield from environmental backgrounds and a cooling system to ensure the required temperature for the NIT detector. A thickness of $40 \mathrm{~cm}$ for the polyethylene blocks and of $10 \mathrm{~cm}$ for the lead plates was chosen to get a negligible contribution from environmental background. The whole structure installed in Hall B is shown in Fig. 3.

\section{Background and sensitivity}

Background sources for dark matter searches are $\alpha$ and $\beta$ particles, $\gamma$-rays and neutron induced recoils, while NIT are essentially insensitive to minimum ionizing particles (MIP). The main sources of $\alpha$-particles are $\mathrm{U}$ and Th radioactive chains and Radon. These $\alpha$-particles have MeV energies and their range in emulsion is a few tens of microns, by far longer than WIMP-induced nuclear recoils. $\alpha$-particles can therefore be identified and discarded in the emulsions by an upper cut on the track length. The $\beta$-rays produced in ${ }^{14} \mathrm{C}$ decay can be discarded by using a cryogenic environment [12]. Neutron induced recoils are not distinguishable from the expected WIMP signal, except for the isotropic angular distribution and for the typical track length, largely exceeding the range expected for WIMP-induced recoils. While the external neutron flux can be reduced to a reasonable level with an appropriate shield, the intrinsic emulsion radioactivity would be responsible of an irreducible neutron yield through $(\alpha, \mathrm{n})$ and ${ }^{238} \mathrm{U}$ spontaneous fission reaction. The detectable neutron-induced background has been measured to be about 0.06 per year per kilogram [13], thus allowing to design a detector for an exposure of $10 \mathrm{~kg}$ year without any further purification. 


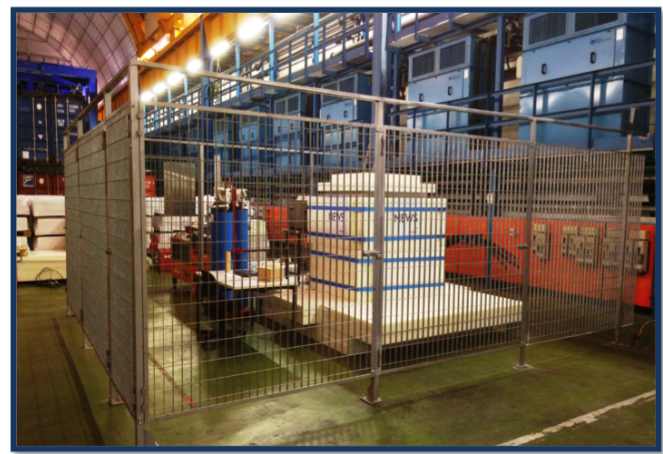

Figure 3. Picture of the shielded structure installed in the underground Hall B at Gran Sasso.

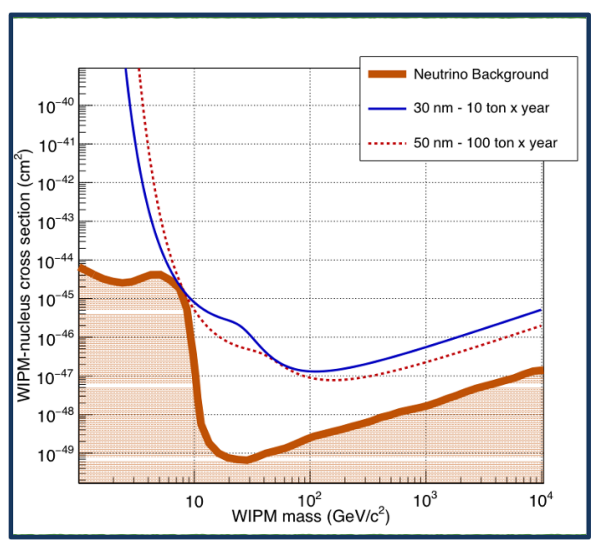

Figure 4. Discovery potential of an emulsion detector with different track length thresholds and exposures.

Fig. 4 shows the discovery potential of the NEWSdm experiment [2]. Different track length thresholds and detector exposures are assumed: the dotted red line represents a $50 \mathrm{~nm}$ threshold with 100 ton year exposure while the solid blue line assumes a $30 \mathrm{~nm}$ threshold with an exposure of 10 ton year. Both curves assume a negligible background. The presence in the emulsions of lighter nuclei such as carbon in addition to the heavier nuclei of silver and bromine results in a good sensitivity to WIMPs in the mass range between 10 to $100 \mathrm{GeV} / \mathrm{c}^{2}$. We have also compared the exclusion limits with the thick orange curve representing the so-called neutrino floor for a Xe/Ge detector [14]. Given the nanometric accuracy achieved, it is realistic to assume a position resolution of several tens of nanometers provided that the grain density is sufficiently high. A mass of about 10 ton would provide sensitivity to explore the region beyond the neutrino floor.

\section{Summary and plans}

NEWSdm aims at providing an unambiguous signature of the Galactic dark matter and at extending dark matter searches beyond the neutrino floor. The directionality has been proven with Carbon ions of the energies relevant for dark matter searches. The current intrinsic radioactive level allows to design an experiment with a $10 \mathrm{~kg}$ year exposure. The selection of the emulsion components could further increase the radiopurity, thus allowing larger exposures. We plan a pilot experiment with $10 \mathrm{~kg}$ year exposure and explore the region indicated by the DAMA experiment with a powerful and complementary approach, acting also as a demonstrator of this new directional technique.

\section{References}

[1] Battat J B R et al, Physics Reports 662 (2016) 1

[2] Agafonova N et al, NEWSdm Collaboration, Eur. Phys. J. C78 (2018) no.7, 578

[3] Natsume N et al, Nucl. Instr. Meth. A 575 (2007) 439

[4] Naka T et al, Nucl. Instrum. Meth. A 718 (2013) 519

[5] Agafonova A et al, OPERA Collaboration, JINST 4 (2009) P04018.

[6] Alexandrov A et al, JINST 10 (2015) P11006 
[7] Alexandrov A et al, JINST 11 (2016) P06002

[8] Alexandrov A et al, Nature Scientific Reports 7 (2017) 7310

[9] Naka T et al, EAS Publ. Ser. 53 (2012) 51

[10] Kimura M and Naka T, Nucl. Instrum. Meth. A 680 (2012) 12

[11] Tamaru H et al, Applied Phys. Lett. 80 (2002) 1826

[12] Kimura M et al, Nucl. Instrum. Meth. A845 (2017) 373-377.

[13] Alexandrov A et al, Astropart. Phys. 80 (2016) 16

[14] Billard J, Strigari L and Figueroa-Feliciano E, Phys. Rev. D 89 (2014) 2 